
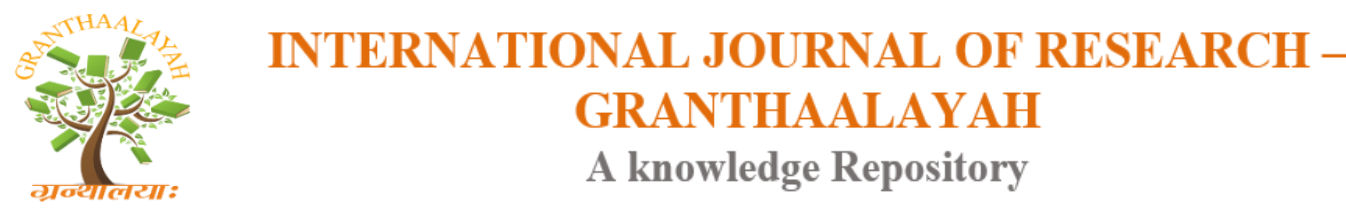

Science

\title{
CONTENT BASED IMAGE RETRIEVAL SYSTEM BY FUSION OF COLOR, TEXTURE AND EDGE FEATURES WITH SVM CLASSIFIER AND RELEVANCE FEEDBACK
}

\author{
Priyanka Saxena ${ }^{* 1}$, Shefali ${ }^{2}$ \\ ${ }^{*}$ PG Scholar, Electronics and Communication Department, Kurukshetra University, \\ Kurukshetra, India, \\ ${ }^{2}$ Assistant professor, Electronics and Communication Department, Kurukshetra University, \\ Kurukshetra, India
}

\begin{abstract}
Content Based Image Retrieval system automatically retrieves the most relevant images to the query image by extracting the visual features instead of keywords from images. Over the years, several researches have been conducted in this field but the system still faces the challenge of semantic gap and subjectivity of human perception. This paper proposes the extraction of lowlevel visual features by employing color moment, Local Binary Pattern and Canny Edge Detection techniques for extracting color, texture and edge features respectively. The combination of these features is used in conjunction with Support Vector Machine to reduce the retrieval time and improve the overall precision. Also, the challenge of semantic gap between low and high level features is addressed by incorporating Relevance Feedback. Average precision value of 0.782 was obtained by combining the color, texture and edge features, 0.896 was obtained by using combined features with SVM, 0.882 was obtained by using combined features with Relevance Feedback to overcome the challenge of semantic gap. Experimental results exhibit improved performance than other state of the art techniques.
\end{abstract}

Keywords: Retrieval System; Fusion; Features; Relevance Feedback.

Cite This Article: Priyanka Saxena, and Shefali. (2018). "CONTENT BASED IMAGE RETRIEVAL SYSTEM BY FUSION OF COLOR, TEXTURE AND EDGE FEATURES WITH SVM CLASSIFIER AND RELEVANCE FEEDBACK." International Journal of Research Granthaalayah, 6(9), 259-273. https://doi.org/10.5281/zenodo.1443433.

\section{Introduction}

The growth of internet technology has witnessed an enormous increment in the number of digital images worldwide available on the internet. Users demand an efficient image retrieval system that will not only resolve the problem of manually annotating and entering the keywords but at the same time reduce the retrieval time. Over the years, text based image retrieval systems were used for retrieving images from a huge database that closely resemble the query image input by the user. 
This image retrieval technique has proved to be cumbersome for very large databases such as geospatial satellite imagery, medical databases where the size of database is quite large and the keywords are difficult to memorize. The problem of semantic gap also exist in text based image retrieval systems. It sometimes becomes impossible to manually annotate images in a very large database. The challenges faced by conventional image retrieval systems encouraged the researchers to overcome the challenges by designing an automatic retrieval system that uses the visual features instead of keywords for retrieving images relevant to the query. Content Based Image Retrieval (CBIR) system retrieves the images that are most relevant to the query image from an image database by extracting the low level visual features such as color, texture, shape using appropriate retrieval techniques. In 1992 T. Kato used the term content-based image retrieval to describe the experiments into retrieving the images automatically from a database by using color and shape feature extraction.

The earliest CBIR system popularly known as QBIC was developed by IBM. The various commercial CBIR systems are QBIC, Virage, VisualSEEK, Netra, Photobook and SIMPLIcity. CBIR system involves two basic steps. The first step extracts the low level visual features from the query and database images and the second step computes similarity of the feature vectors obtained from the first step. CBIR system finds applications in fields such as Medical diagnosis, Geospatial Satellite Imagery, Fashion Designing Crime Prevention, Face recognition, Architectural and Engineering design, Textile industry and others. The current CBIR systems are still lack in accuracy of relevant image due to the improper selection of feature extraction methods and similarity measurement. Gap between information extracted automatically from the visual data and interpretation by the user which is known as Semantic gap. Subjectivity of human perception of visual content.

\subsection{Basic CBIR System}

First, the Query Image is input by the user. Low level features such as color, texture and shape are extracted from the query image as well as database images. Feature vector is formed from the low level features extracted. The feature vectors of database images are stored in a binary file. The similarity between query image and database images is computed to obtain the most relevant images from the database. The database images are arranged in ascending order corresponding to their similarity evaluation. The block diagram of basic CBIR system is shown in Figure 1.

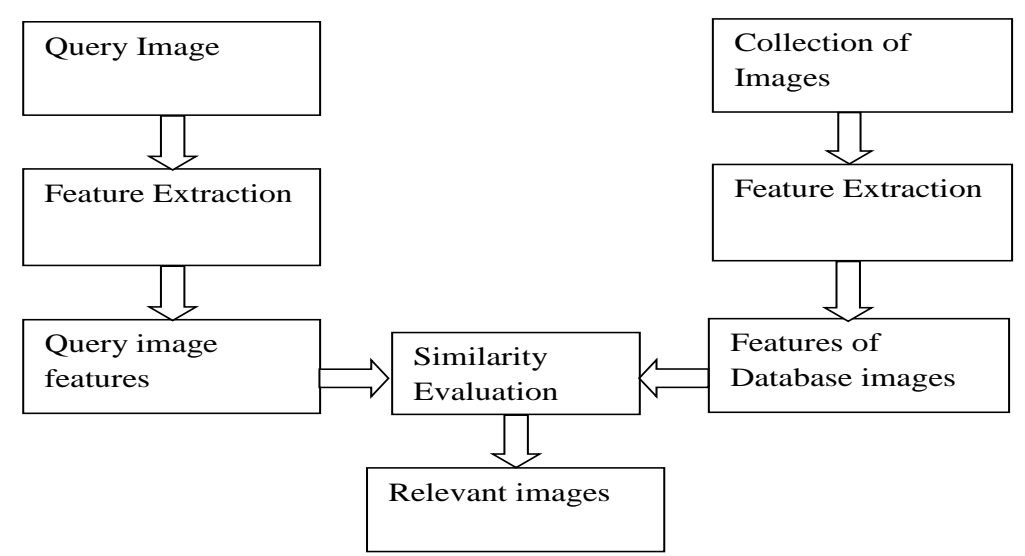

Figure 1: Block diagram of CBIR 


\section{Literature Survey}

A CBIR system based on Color strings comparison was proposed. Database was classified using Support Vector Machine Classifier in order to obtain different classes. Features of the query image and for all images in the specified class label are extracted using color string coding and comparison method. Database classification improves the performance and color string coding gives better results [1].

A hybrid feature based efficient CBIR system was proposed using various distance metrics. Spatial domain features such as color auto-correlogram, color moments, HSV histogram features and frequency domain features such as moments using SWT and Gabor wavelet transform were used. The precision was improved by using binarized statistical image features, Color and Edge directivity descriptor features. WANG database containing 1000 images was used. Similarity measurement was done using Euclidean distance, City block distance, Minkowski distance and Malabonis distance. High precision was achieved by using BSIF and CEDD descriptor [2].

A novel similarity evaluation using a meta-heuristic algorithm called a memetic algorithm (genetic algorithm with great deluge) is achieved between the features of the QI and the features of the database images. This work proposed an effective CBIR system using MA to retrieve images from databases. Then, using the MA based similarity measure; images that are relevant to the QI were retrieved efficiently. Corel image database was used. It has strong capability to discriminate color, shape and color, texture features. The results outperformed other CBIR systems in terms of precision and recall where average precision and recall rates were 0.882 and 0.7002 respectively $[7]$.

The overview of various techniques used for extracting low level features i.e. color and Texture was given. Color feature extraction techniques are Color histogram, color Correlogram, color cooccurance matrix and Dominant Color descriptor. Texture feature extraction techniques are Tamura teaxture feature, steerable pyramid, wavelet Transform, Gabor wavelet Transform. A comparative analysis of color and texture feature extraction techniques wiith their advantages and disadvantages was given [12].

A CBIR system based on Interactive Genetic Algorithm was proposed to increase the accuracy of image retrieval. The retrieval process was split into two stages. In the query stage, the query features were extracted and the similarity between the query image and database image features was evaluated. In the evolution stage, the most relevant images were retrieved using Interactive Genetic Algorithm. The proposed system incorporates user oriented CBIR that uses Interactive Genetic Algorithm to find the most relevant images to the user. IGA provides an interactive mechanism to effectively capture the user's intention [16].

Color feature was extracted by color moments, texture feature was extracted by wavelet and shape feature was extracted by using Edge Histogram. The classification of image into different groups is performed by incorporating SVM classifier and the computation of similarity is carried out within the same class. The combination of color, texture and shape feature exhibits higher values of average precision compared to individual features [41]. 
A content Based image retrieval system using Support vector machine based Relevance Feedback that incorporates not only positive but also negative feedbacks for retrieving images is proposed. SVM learns the information regarding the positive and negative examples and the desired weights are automatically updated for images that are positive and relevant. Results show better performance [50].

\section{Proposed Methodology}

A CBIR using hybrid framework is developed in which the images relevant to query image are extracted by carrying out color, texture and edge feature extraction by employing color moment, Local Binary Pattern and Canny edge detection techniques respectively. The similarity between features of query image and selected images is then computed by using Manhattan distance. Bubble sort algorithm is used to sort the images. Support vector machine is used after combining the feature vectors obtained by extracting color, texture and edge using appropriate weights.

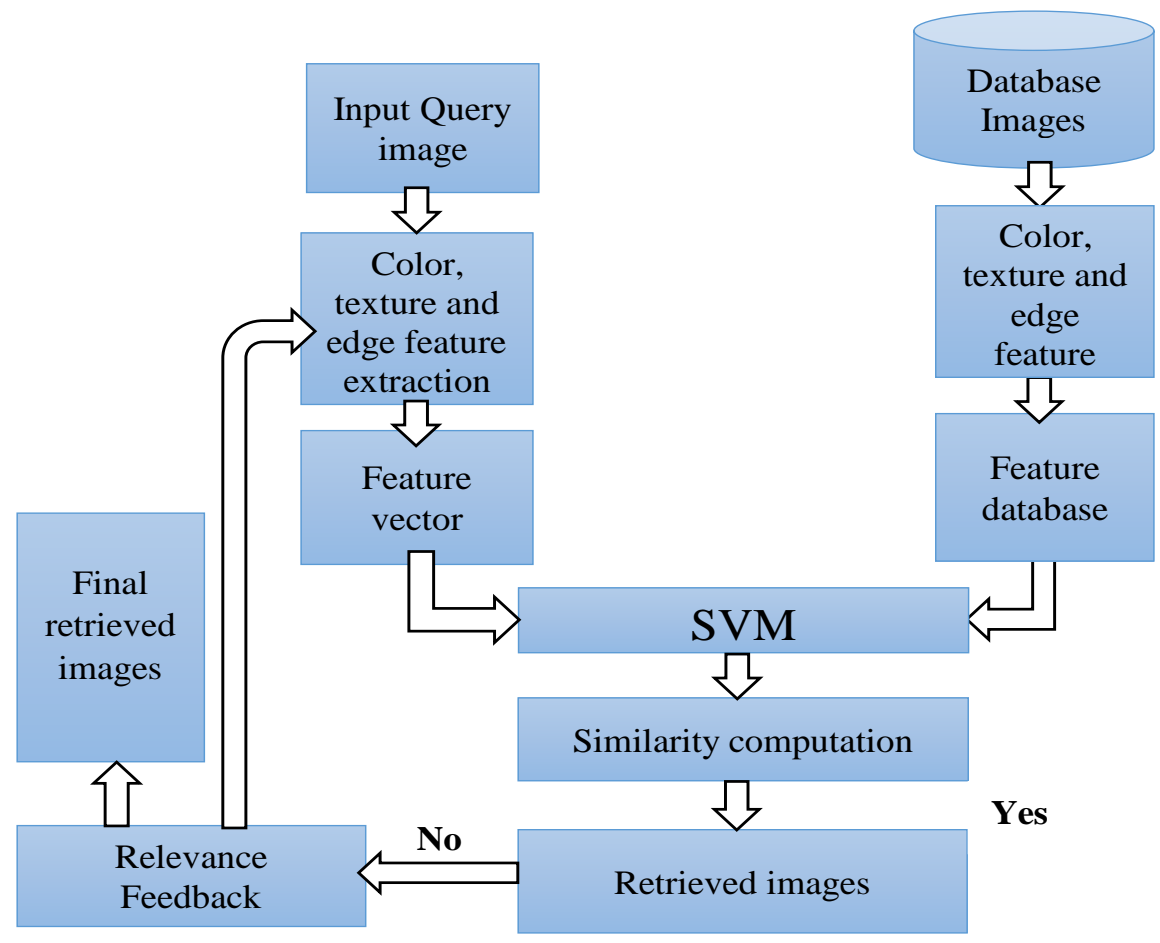

Figure 2: Block diagram of proposed system

Finally the problem of semantic gap is overcome by using relevance feedback that allows the user to select relevant and irrelevant images as per his/her requirement. The Block diagram of proposed CBIR system is shown in Fig 2.

\subsection{Wang's Database}

Wang's database is a database of 1000 images belonging to ten categories namely Africa, Sea, Buildings, Buses, Dinosaur, Elephants, Flowers, Horses, Mountains and Food as shown in Figure 3. Each category comprises of 100 images. 
1. Africa

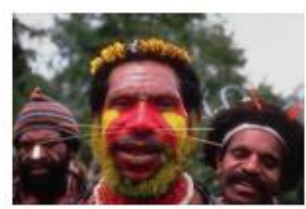

2. Sea

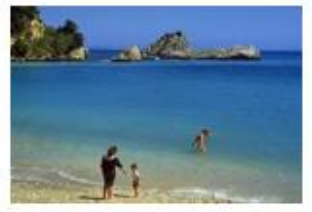

3. Buildings

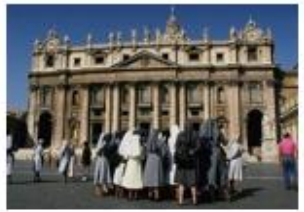

4. Buses

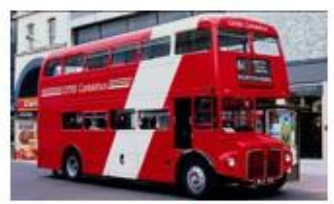

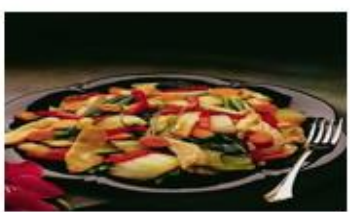

10. Food
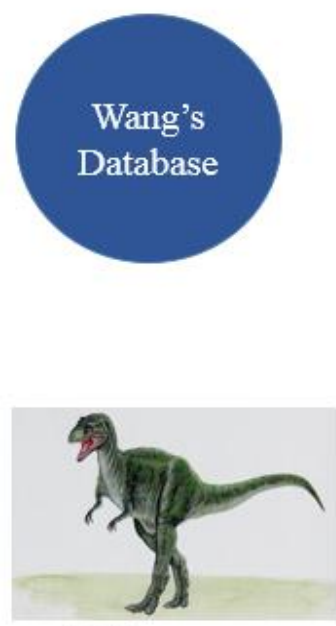

5. Dinosaur

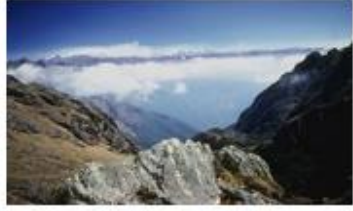

9. Mountains

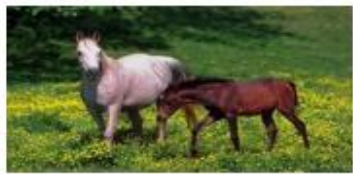

8. Horses

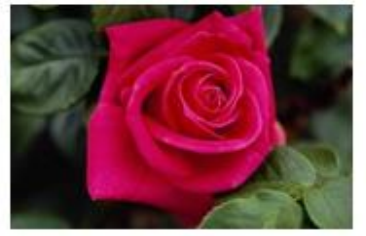

7. Flowers

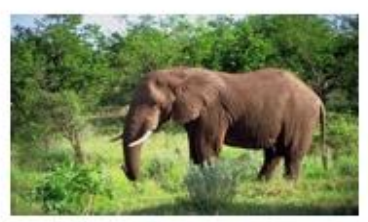

6. Elephants

Figure 3: Wang's Database

\subsection{Color Feature Extraction Using Color Moments}

First order moment: It describes the average information about pixel distribution of a given image.

Second order moment: It describes the closeness of pixel distribution about mean color.

Mean (Ic) $=\frac{1}{M x N} \sum_{i=1}^{M} \sum_{j=1}^{N} P c i j, \mathrm{c}=[\mathrm{R}, \mathrm{G}, \mathrm{B}]$

$\mathrm{Ic}=$ color channel information

$\mathrm{M}, \mathrm{N}=$ row and column size of image

Pcij=image pixel value in ith row and jth column

$\operatorname{Std}\left(I_{c}\right)=\left(\frac{1}{M x N} \sum_{i=1}^{M} \sum_{j=1}^{N}\left(P_{c i j}-\operatorname{Mean}\left(I_{c}\right)\right)^{2}\right)^{\frac{1}{2}}, \mathrm{c}=[\mathrm{R}, \mathrm{G}, \mathrm{B}]$

\subsection{Texture Feature extraction using Local Binary Pattern}

The images in RGB color space are converted into gray scale before carrying out actual processing. In each iteration the input is a $3 \times 3$ gray scale image. The threshold for pixels in the neighborhood is obtained by using the center position pixel of the sub-block. The sub-block is represented using the binary values obtained from thresholding where LBP estimation for $3 \times 3$ sub-block is performed using the following equation. 


$$
\mathrm{LBP}_{N}=\sum_{i=0}^{N-1} f\left(P_{i}-\mathrm{CP}\right) 2^{i} ; \mathrm{f}(\mathrm{p})=\left\{\begin{array}{l}
1 ; P \geq 0 \\
0 ; P<0
\end{array}\right.
$$

$\mathrm{N}=$ No. of neighboring pixels for center pixel in $3 \times 3$ sub-block

\subsection{Edge Features Extraction Using Canny Edge Detector}

Edges are formed by abrupt change in intensity. A multi stage algorithm is employed for wide range edge detection in images. Edge detection is perfect, clear response and good image localization is achieved. Gaussian Blur is used to remove Speckles and image noise. Gradient's intensity and direction is obtained. Non-max repression determines if a pixel is a better candidate for edge than its neighbors.

\subsection{Support Vector Machine}

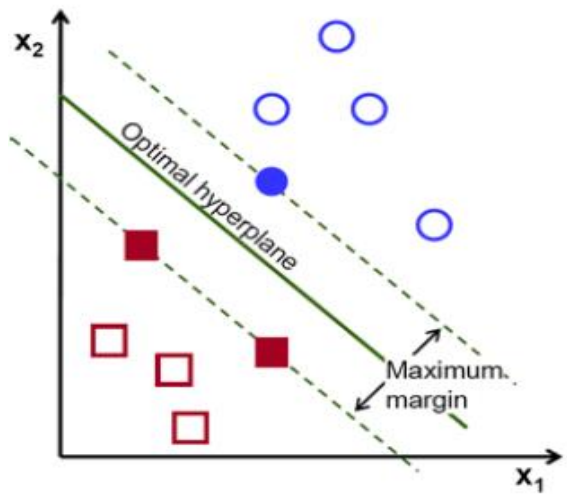

Figure 4: Support Vector Machine Hyperplane

The training vectors of two classes is represented in the form of a set. The problem is to differentiate between these two classes

$$
\left\{\left(\overrightarrow{x_{l}}, y_{j}\right)\right\}_{i=1}^{N}, \quad y_{i}=+1 /-1
$$

$x_{i}=$ pattern given as input, $y_{j}=$ label

+1 indicates that an example is positive while -1 indicates that the example is negative. The hyperplane that differentiates the two classes is given as:

$$
\vec{w}^{T} \vec{x}+\mathrm{b}=0
$$

$\vec{x}=$ vector input, $\vec{w}=$ weight vector, $\mathrm{b}=$ bias

For obtaining an optimal hyperplane the values $w_{o}$ and $b_{o}$ need to be found for maximizing the separation between hyperplane and closest data points.

$$
w^{T} \overrightarrow{x_{l}}+b_{o} \geq 1 \quad \text { for } y_{i}=+1
$$


$w^{T} \overrightarrow{x_{\imath}}+b_{o} \geq-1 \quad$ for $y_{i}=-1$

The separation between a point $\vec{x}$ and optimal hyperplane is given by:

$$
d\left(w_{o}, b_{o}, \vec{x}\right)=\frac{\left|w_{o}^{T} \vec{x}+b_{o}\right|}{\left\|w_{o}\right\|}
$$

\subsection{Relevance Feedback}

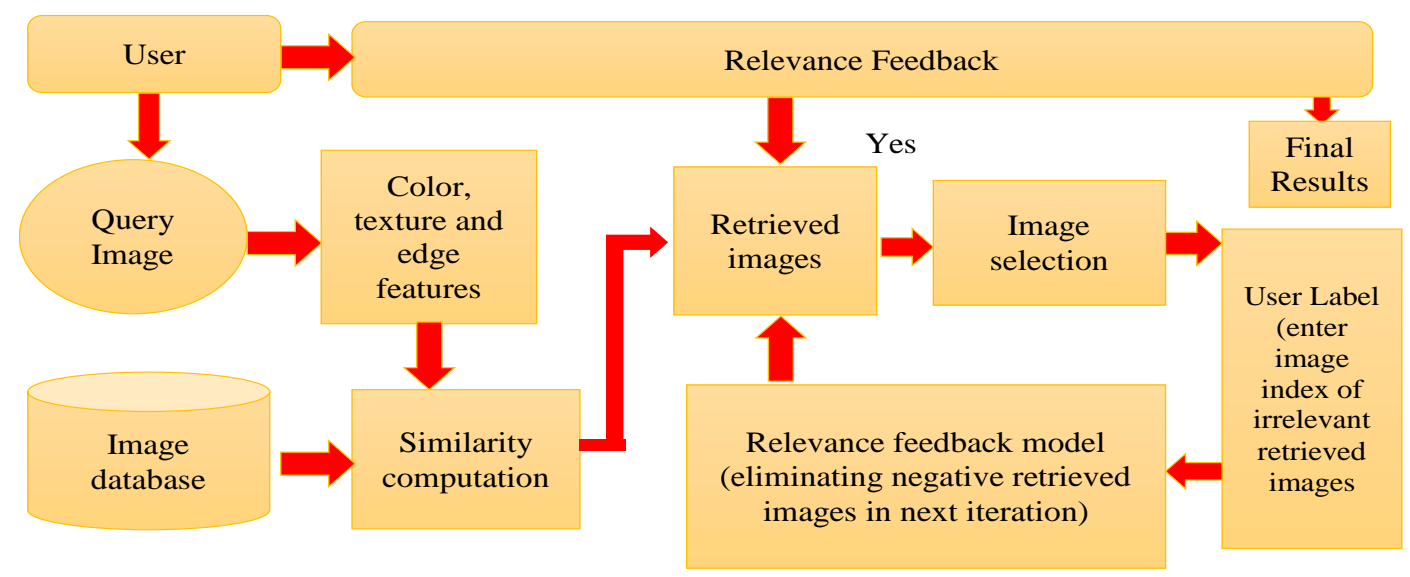

Figure 5: Block diagram of relevance feedback

Relevance feedback involves the user for selecting more semantically relevant images to the query. When any image is input by the user its low level features are extracted. Similarity metric is used for sorting the most relevant images. If the retrieved images satisfy the user, the process of retrieval is halted. If the retrieved images do not meet the user requirement the index numbers of the irrelevant images are input by the user. These images are rejected for the next iteration and the similarity metric is recalculated. Sorting is performed based on the similarity value. If the satisfaction of the user is still not met the user again inputs the index of irrelevant images and relevance feedback process is repeated. The block diagram of proposed Relevance feedback is shown in Figure 5.

\subsection{Algorithm}

Algorithm for content based image retrieval system by fusion of color, texture and edge features with Support Vector machine and Relevance Feedback

1) The R, G, B color channels extracted from the colored images are separated.

2) The visual features namely, color, texture and edge are extracted by incorporating color moments, Local Binary Pattern and Canny edge detection techniques.

3) The feature vectors obtained by extracting color, texture and edge features are combined.

4) The similarity between the query image and images from the database is computed using the Manhattan distance.

5) SVM classifier is used to identify the class to which the query image belongs and the images relevant to the query from that class are retrieved. 
6) If the user is satisfied with the retrieved images the final results are displayed.

7) If the user is not satisfied with the results the image indexes of the irrelevant images are entered in the next iteration.

8) These irrelevant images are eliminated in the next iteration.

9) Relevance feedback is finally used to iteratively refine the results to obtain the most relevant images as per the user's requirement.

\section{Experimental Results}

In this paper, the extraction of images relevant to the user's query was performed by extracting three visual features namely color, texture and edge from the query image as well as images belonging to the Wang's database. The Wang's database comprises of 1000 images divided into 10 categories. Each category consists of 100 images. Color feature extraction was done by employing Color moments, texture feature extraction using Local Binary Pattern and edge feature extraction using canny edge detection technique.

The user inputs a query image whose low level features (color, texture and edge) are extracted using color moments, Local Binary Pattern and Canny edge detection techniques respectively. The precision recall and f-measure value of random images input by the user from each category of the Wang's database is tabulated in table and a graph of precision, recall and f-measure values obtained by color feature extraction using color moments for 10 categories is plotted. Figure 6 a. shows a sample query image input by the user and corresponding top ten retrieved images from the database that are most similar to the query image extracted using color moments. The Manhattan distance that indicates the similarity between the retrieved images from database and query image is displayed on the top of each retrieved image. Top 10 images that are nearest to the query image are retrieved. Lesser the value of Manhattan distance of retrieved image higher the similarity with query image. Further, Figure 6. b. shows a sample query image from the category flowers of the Wang's database and the corresponding top 10 images retrieved from the database by extracting the texture features from query and database images using Local binary pattern.

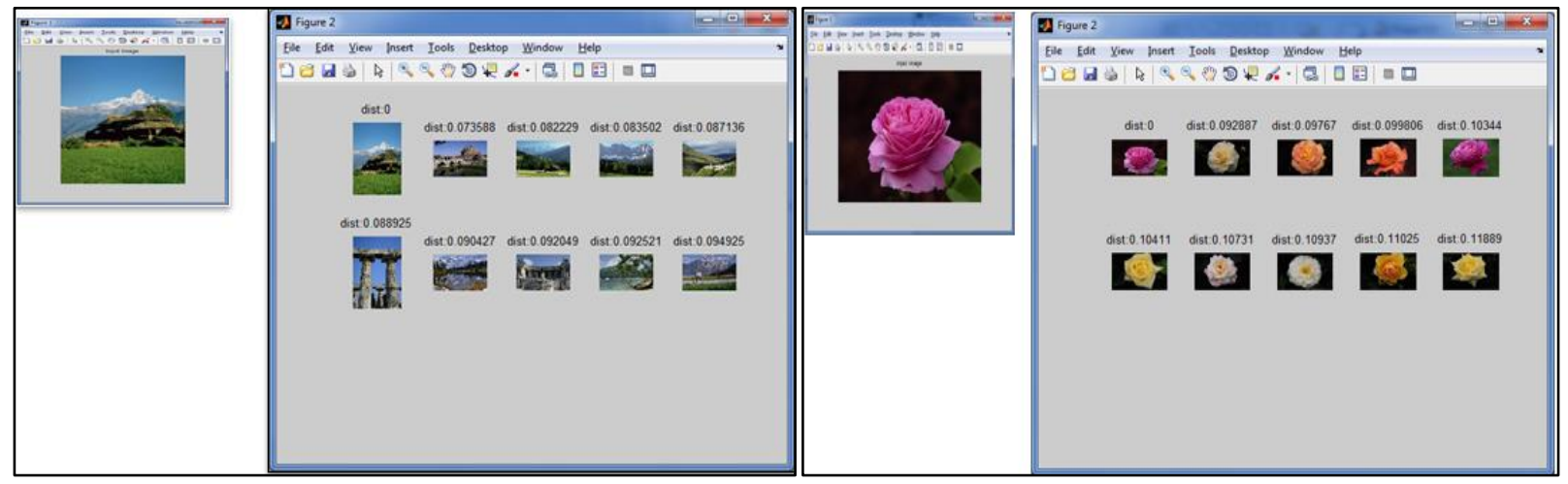

Figure 6a: Images Retrieved by extracting color features using Color moments b. Images Retrieved by extracting texture features using Local Binary Pattern

Figure 7. a. shows a sample query image from the category sea of the Wang's database and the corresponding top 10 images retrieved from the database by extracting the edge features from query and database images using Canny edge detection technique. The Manhattan distance is 
displayed on the top of each retrieved image. Figure 7. b. shows a sample query image from the category flowers of the Wang's database and the corresponding top 10 images retrieved from the database by extracting the combined color, texture and edge features from query and database images using Color moments, LBP and Canny edge detection techniques.
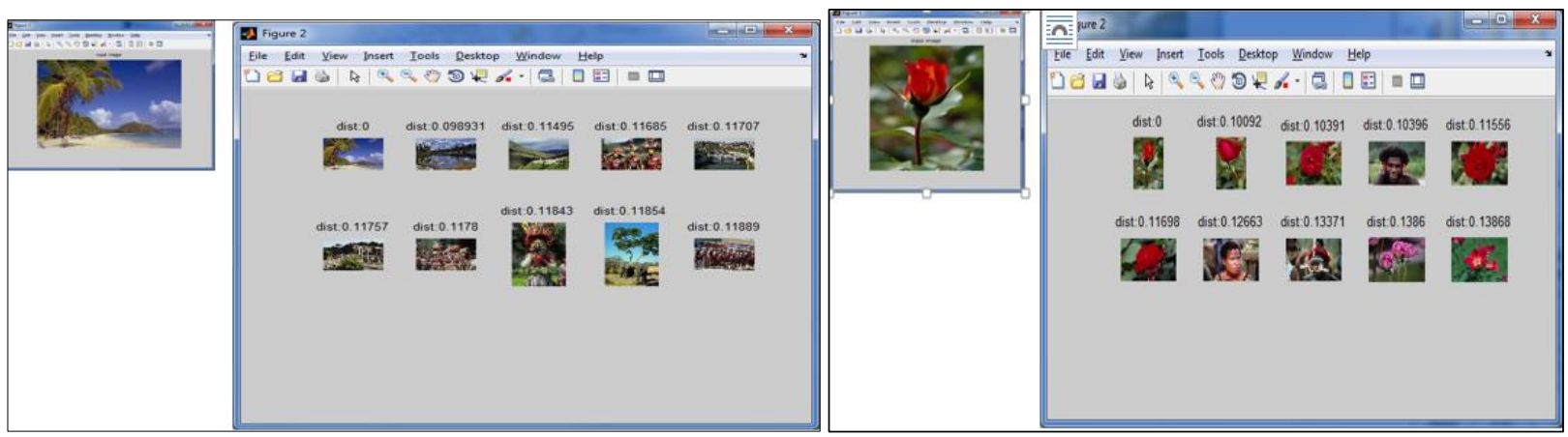

Figure 7a: Images Retrieved by extracting edge features using Canny Edge Detection. b. Images Retrieved by extracting combined (color, texture, edge) features

Figure 8. a. shows the top 10 retrieved images similar to the query image belonging to the category mountain of the Wang's database. Images are retrieved by extracting combined color, texture and edge features. Further, Figure 8 . b. shows the top 10 retrieved images similar to the query image belonging to the category mountain of the Wang's database. Images are retrieved by extracting combined color, texture and edge features and using Support Vector Machine classifier.

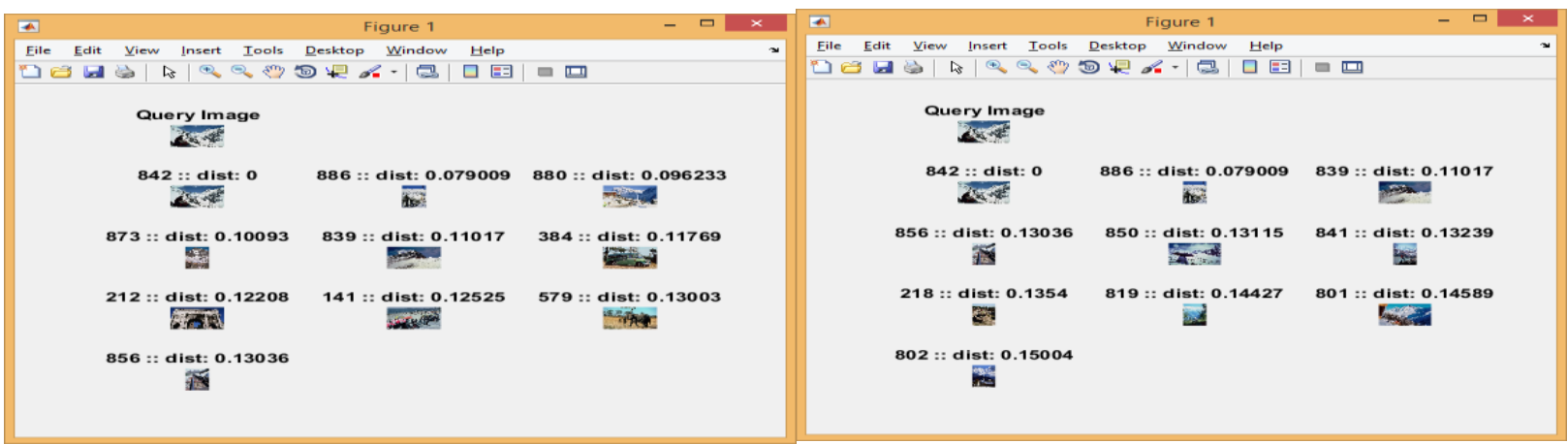

Figure 8a: Retrieved images extracted by combining color, texture and edge features. b. Retrieved images extracted by combining color, texture and edge features using SVM

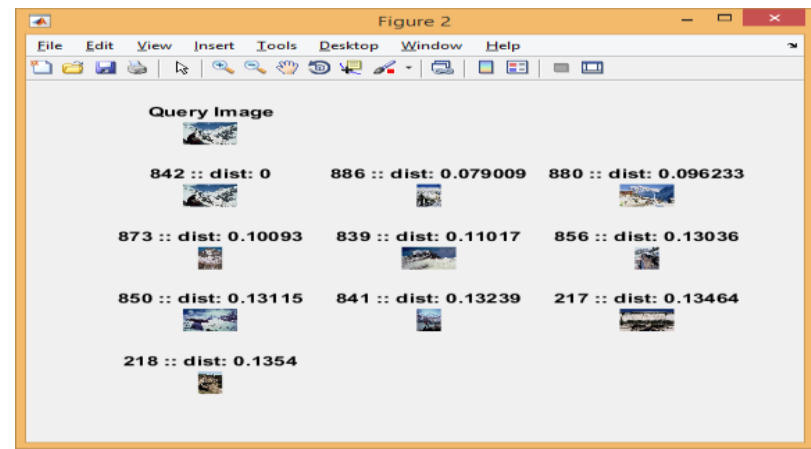

Figure 9: Retrieved images extracted by combining color, texture and edge features and Relevance Feedback. 
Further, figure 9. Shows the top 10 retrieved images similar to the query image belonging to the category mountain of the Wang's database. Images are retrieved by extracting combined color, texture and edge features and relevance feedback is incorporated to refine the retrieval results. The precision, recall and f-measure values for images retrieved in fig 8.a, 8.b. and 9 for combined features, combined features with SVM and combined features with relevance feedback have been tabulated in table 1.

Table 1: Precision, Recall and F-measure for Fig 8. a., 8.b. and 9 for combined features, combined features with SVM and combined features with relevance feedback

\begin{tabular}{|l|l|l|l|}
\hline $\begin{array}{l}\text { Performance } \\
\text { Parameters }\end{array}$ & $\begin{array}{l}\text { Combined } \\
\text { features }\end{array}$ & $\begin{array}{l}\text { Combined features } \\
\text { with SVM }\end{array}$ & $\begin{array}{l}\text { Combined features with } \\
\text { Relevance Feedback }\end{array}$ \\
\hline Precision & 0.6 & 0.9 & 0.8 \\
\hline Recall & 0.06 & 0.09 & 0.08 \\
\hline Fmeasure & 0.109 & 0.1636 & 0.1454 \\
\hline
\end{tabular}

The average precision value of five random images input by the user from each category of the Wang's database by extracting color texture and edge features individually and then combining them is tabulated in table 2 .

Table 2: Comparison of precision values obtained for images belonging to Wang's database by extracting color texture and edge features individually and then combining them

\begin{tabular}{|l|l|l|l|l|l|}
\hline Category & $\begin{array}{l}\text { Color } \\
\text { Moments }\end{array}$ & $\begin{array}{l}\text { LBP } \\
\text { texture }\end{array}$ & $\begin{array}{l}\text { Canny } \\
\text { Edge }\end{array}$ & $\begin{array}{l}\text { L.K. Pavithra et } \\
\text { al. }\end{array}$ & Combined \\
\hline Africa & 0.62 & 0.68 & 0.4 & 0.73 & 0.82 \\
\hline Sea & 0.54 & 0.52 & 0.22 & 0.82 & 0.8 \\
\hline Buildings & 0.38 & 0.76 & 0.3 & 0.75 & 0.72 \\
\hline Bus & 0.42 & 0.8 & 0.84 & 0.87 & 0.94 \\
\hline Dinosaurs & 1 & 0.96 & 0.58 & 0.93 & 1 \\
\hline Elephants & 0.4 & 0.38 & 0.4 & 0.76 & 0.62 \\
\hline Flowers & 0.68 & 1 & 1 & 1 & 0.86 \\
\hline Horse & 0.92 & 0.8 & 0.76 & 0.82 & 0.91 \\
\hline Mountains & 0.34 & 0.26 & 0.46 & 0.8 & 0.44 \\
\hline Food & 0.52 & 0.56 & 0.52 & 0.71 & 0.71 \\
\hline Average & 0.582 & 0.672 & 0.548 & 0.832 & 0.782 \\
\hline
\end{tabular}

Comparison of precision values obtained for images belonging to Wang's database by extracting combined color texture and edge features with SVM and without SVM and combined features with Relevance Feedback has been tabulated. The average precision value of five random images input by the user from each category of the Wang's database is tabulated in table 3. 
Table 3: Comparison of precision values obtained for images belonging to Wang's database by extracting combined color texture and edge features with SVM and without SVM and combined features with Relevance Feedback

\begin{tabular}{|l|l|l|l|l|}
\hline Category & $\begin{array}{l}\text { L.K. } \\
\text { Pavithra } \\
\text { al. }\end{array}$ & $\begin{array}{l}\text { Combined } \\
\text { features }\end{array}$ & $\begin{array}{l}\text { combined features } \\
\text { with SVM }\end{array}$ & $\begin{array}{l}\text { Combined features with } \\
\text { RF (without SVM) }\end{array}$ \\
\hline Africa & 0.73 & 0.82 & 0.8 & 0.96 \\
\hline Sea & 0.82 & 0.8 & 0.82 & 0.76 \\
\hline Buildings & 0.75 & 0.72 & 0.9 & 0.8 \\
\hline Bus & 0.87 & 0.94 & 1 & 0.83 \\
\hline Dinosaurs & 0.93 & 1 & 1 & 1 \\
\hline Elephants & 0.76 & 0.62 & 0.98 & 0.84 \\
\hline Flowers & 1 & 0.86 & 1 & 1 \\
\hline Horse & 0.82 & 0.91 & 0.8 & 1 \\
\hline Mountains & 0.8 & 0.44 & 0.66 & 0.77 \\
\hline Food & 0.71 & 0.71 & 1 & 0.86 \\
\hline Average & 0.832 & 0.782 & 0.896 & 0.882 \\
\hline
\end{tabular}

A graph of precision values obtained by color, texture, edge feature extraction, for 10 categories is plotted as shown in Figure 10.

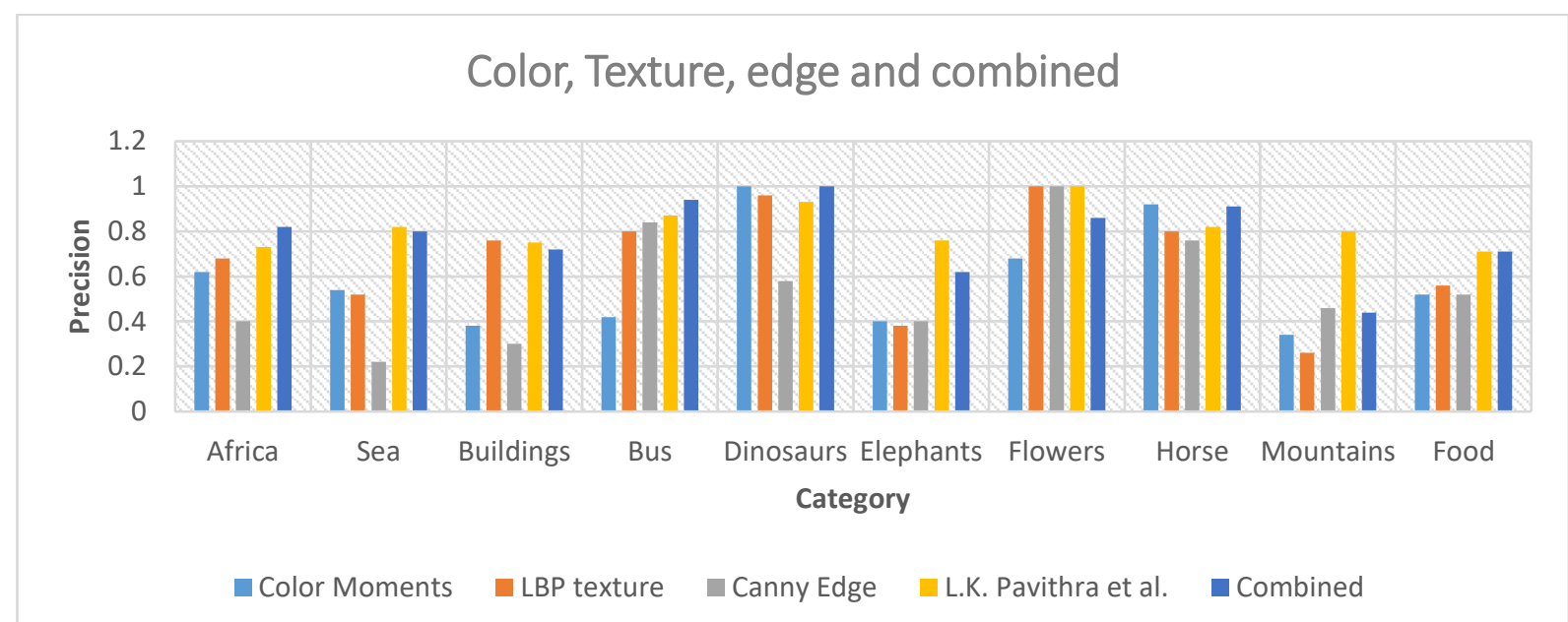

Figure 10: Graph for Comparison of precision values obtained for images belonging to Wang's database by extracting color texture and edge features individually and then combining them

A graph of precision values obtained by combined color, texture, edge feature extraction, combined feature with SVM and Combined feature with relevance feedback for 10 categories is plotted as shown in Figure 11. 


\section{Results}

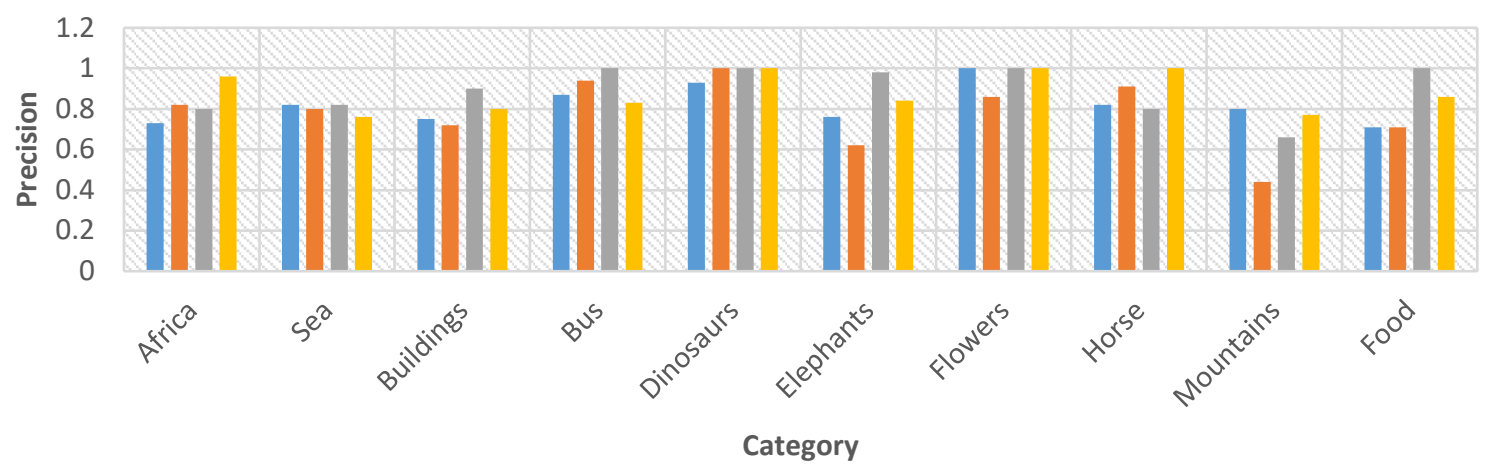

- L.K. Pavithra et al. $\quad$ Combined combined features with SVM $\quad$ Combined features with RF

Figure 11: Graph for Comparison of precision values obtained for images belonging to Wang's database by extracting combined color texture and edge features with SVM and without SVM and combined features with Relevance Feedback

\section{Conclusion}

In this paper, the extraction of images relevant to the user's query was performed by extracting three visual features namely color, texture and edge from the query image as well as images belonging to the Wang's database. The performance of CBIR system by extracting individual features only is not effective while their combination yields good results. Color, texture and edge feature extraction was done by employing Color moments, Local Binary Pattern and Canny edge detection techniques respectively. Analysis is carried out by first retrieving images using color, texture and edge features individually, then by combining them and finally by applying SVM. Relevance feedback has been used to overcome the semantic gap. Results show that the performance of proposed system exhibits high precision value than other state of art techniques.

\section{References}

[1] K. Jenni, S. Mandala \& M.S. sunar, "Content Based Image Retrieval using Color String Comparison,” Elsevier, Procedia Computer Science, Vol. 50, pp. 374-379, 2015.

[2] Y. Mistry, D.T. Ingole \& M.D. Ingole, "Content based image retrieval using hybrid features and various distance metric," Journal of Electric Systems and Information Technology, vol. 18, pp. 335-342, 2016

[3] Hassan Farsi, Sajad Mohammadzadeh, "Color and Texture based image retrieval using Hadamard matrix in discrete wavelet transform," IET Image Processing 2013, vol 7, Iss 3, pp 212-218 doi: 10.1049/iet-ipr.20122.0203,2013

[4] I.J.Sumana, M.M.Islam \& D.Zhang, "Content based image retrieval using curvelet transform," Proc. IEEE 10 ${ }^{\text {th }}$ workshop on Multimedia Signal Processing, pp.11-16, 2008.

[5] K. Seetharaman,S.Sathiamoorthy,"Color image retrieval using statistical model and radial basis function neural network", Egyptian Informatics Journal ,2014 
[6] Xiang-Yang Wang, Hong-Ying Yang, Dong-Ming Li, "A new content-based image retrieval technique using color and texture information", Elsevier, doi.org/10.1016/j.compeleceng.2013.01.005, 2013.

[7] M. K. Alsmadi, "An efficient similarity measure for content based image retrieval using memetic algorithm," Egyptian Journal of Basic and Applied Sciences, Vol. 4, pp- 112- 122, 2017.

[8] Heba Aboulmagd, Neamat El-Gayar, Hoda Onsi, "A new approach in content base image retrieval using fuzzy", Springer, Doi 10.1007/s 1235-008-9142.

[9] Kashif Iqbal, Michael O.Odetayo, Anne James, "Content based image retrieval approach for biometric security using color, texture and shape features controlled by fuzzy heuristics", Journal of computer and System Sciences, doi:10.1016/j.jcss.2011.10.013, pp 1258-1277.

[10] B.Verma, S.Kulkarni, "A fuzzy-neural approach for interpretation and fusion of color and texture features for CBIR system”,Elsevier, doi:10.1016/j.asoc.2004.06.002, 2004.

[11] Jun Yue, Zhenbo Li, Lu Liu, Zetian Fu, "Content based image retrieval using color and texture fused features", Journal of Mathematical and Computer Modelling 54(2011), pp. 11221-11227, 2010.

[12] Jigisha M. Patel, Nikunj C. Gamit, "A Review on Feature Extraction Techniques in Content Based Image Retrieval," Proc. IEEE WiSPNET 2016 conference,2016.

[13] M.Saad,"Low-level color and texture feature extraction for content based image retrieval, "Final Project Report, EE K 381(2008), pp.20-21

[14] K.F. Man, K.S. Tang, S.Kwong, "Genetic Algorithms: Concepts and Applications", IEEE Transactions on industrial electronics, Vol. 43, No. 5, October 1996.

[15] Mutasem K. Alsmadi, "Query-sensitive similarity measure for content based image retrieval using meta-heuristic algorithm", Journal of King Saud University-Computer and Information Sciences (2017), doi 10.1016/2017.05.002, 2017.

[16] M.Venkat Dass, Mohammad Rahmat Ali, Mohammad Mahmood Ali, "Image Retrieval

[17] Using Interactive Genetic Algorithm," Proc. 2014 International Conference on Computational Science and Computational Intelligence, DOI 10.1109/CSCI.2014.44, pp 215-220, 2014.

[18] Marcin Korytkwoski, Leszek rutkowski, Rafal Scherer, "Fast Image Classification by boosting fuzzy classifiers", Journal of Information Sciences 327(2016), doi 10.1016, pp 175-182, 2015.

[19] Showkat Ahmad Dar, Zahid Gulzar Khaki, "Content Based Image Retrieval", IOSR Journal of Computer Engineering, vol. 12, PP 87-92, 2013

[20] Abdolraheem Khader Alhassan, Ali Ahmed Alfaki, "Color and Texture Fusion Based method for content Based Image Retrieval", Proc. International Conference on Communication, Control, Computing and Electronics Engineering (ICCCCEE), Kartoum, Sudan, 2017.

[21] Yolanda Perez Pimentelet al.," A Genetic Algorithm Applied to Content Based Image Retrieval for Natural Scenes Classifications", Proc. Thirteen Mexican International Conference on Artificial Intelligence, DOI 10.1109/MICAI.2014.30, 2014

[22] Nameirakpam Dhanachandra, Khumanthem Manglem and Yambem Jina Chanu," Image Segmentation using K-means Clustering Algorithm and Subtractive Clustering Algorithm", Proc. Eleventh International Multi Conference on Information Processing-2015(IMCIP-2015), doi:10.1016, pp 764-771, 2015

[23] Dr. S.D Ruikar, Rohit S. Kabade, "Content Based Image Retrieval by Combining Feature Vector", Proc. IEEE WiSPNET 2016 Conference, 2016.

[24] Esmat Rashedi, Hossein Nezamabadi-pour, "Improving the Precision of CBIR Systems by Feature Selection Using Binary Search Algorithm", Proc. $16^{\text {th }}$ CSI International Symposium on Artificial Intelligence and Signal Processing (AISP 2012), IEEE, 2012.

[25] Roshi Choudhary, Nikita Raina, Neeshu Choudhary, Rashmi Chauhan," An Integrated Approach to Content Based Image Retrieval", Proc. 2014 International Conference on Advances in Computing, Communications and Informatics (ICACCI), IEEE, 2014

[26] W.Y Ma and B.S Manjunath, "Netra: A Toolbox for navigating large image databases," Proc. IEEE Int. Conf. on Image Proc., 1997. 
[27] J.R. Bach, C.Fuller, A Gupta et al.," The Virage image search engine: An open framework for image management," Proc. SPIE storage and Retrieval for Image and Video databases, 1998.

[28] Yong Rui and Thomas S. Huang and Shih-Fu Chang, "Image Retrieval: Current Techniques, Promising Directions and Open Issues", Journal of Visual Communication and Image Representation 10, pp-39-62., 1999.

[29] Mussarat Yamin, Muhammad Sharif, Sajjad Mohsin, "Use of Low Level Features for Content Based Image Retrieval”, Res.J.Recent.Sci., Vol. 2, pp 65-75, 2013.

[30] I.J.Sumana, M.M.Islam \& D.Zhang, "Content based image retrieval using curvelet transform," Proc. IEEE 10 ${ }^{\text {th }}$ workshop on Multimedia Signal Processing, pp.11-16, 2008.

[31] Anelia Grigorova \& Framcesco G. B. De Natale, "Content Based Image Retrieval by Feature Adaptation and Relevance Feedback," IEEE Transactions on Multimedia, Vol. 9, pp. 225-238, 2007.

[32] S.M Mukane, S.R. Gangaje, D.S. Boremane, "A novel scale and rotation invariant texture retrieval method using fuzzy logic classifier", Journal of Computers and Electrical Engineering, 2014.

[33] Alghamdi, R.A Taileb, M and Ameen,"A new Mutimodal Fusion Method based on Association Rules mining for image retrieval", Proc. 17 ${ }^{\text {th }}$ IEEE Mediterranean Electrotechnical Conference (MELECON), pp 493-499, 2014.

[34] K.Shubhankar Reddy and K.Shreedhar, "Image Retrieval Techniques: A Survey", International Journal of Electronic sand Communication Engineering, Vol. 9, pp 19-27, 2016.

[35] Bikesh Kumar Singh, A.S. Thoke, Keshri Verma, Ankita Chandrakar, "Image Information retrieval from incomplete Queries using Color and Shape Features", Signal and Image Processing: An International Journal (SIPIJ), Vol. 2, pp 213-220, 2011.

[36] Swati Aggarwal, A.K Verma, Nitin Dixit, "Content Based Image Retrieval using Color Edge Detection and Discrete Wavelet Transform", Proc. International Conference on Issues and Challenges in Intelligent Computing Techniques, 2014.

[37] Showkat Ahmad Dar, Zahid Gulzar Khaki, "Content Based Image Retrieval", IOSR Journal of computer engineering, Volume 12, pp 87-92, 2013.

[38] L.K. Pavithra, T.Sree Sharmila, "An efficient framework for image retrieval using Color, Texture and Edge features", Journal of Computers and Electrical Engineering, pp 1-14, 2017.

[39] S.Asha, R. Rajesh Kanna, "A Survey on Content Based Image Retrieval Based on Edge Detection", International Journal of Computer Science and Information Technologies, vol. 5(6), pp 8272-8275, 2014.

[40] Sadegh Fadeil, Rassoul Amirffattahi, Mohammad Reza Ahmadzadeh, "A new content based image retrieval system based on optimized integration of DCD, Wavelet and Curvelet Features", IET Image Processing, pp 1-19, 2017.

[41] K.Meena, Dr.A.Suruliandi, "Local Binary Patterns and its Variants for Face Recognition", International Conference on Recent Trends in Information Techgnology (ICRTIT), pp 782-786, 2011.

[42] Naveen A K, N.K. Narayanan, "Image Retrieval using combination of Color, Texture and Shape Descriptor", Proc. 2016 International Conference on Next Generation Intelligent Systems (ICNGIS), IEEE, 2016.

[43] Anuja Khodaskar, Siddharth Ladhake, "Content Based Image Retrieval Using Quantitative Semantic Features”, Springer, HIMI 2014, Part I, LNCS 8521, pp. 439-448, 2014.

[44] Aasia Ali, Sanjay Sharma, "Content Based Image Retrieval using Feature Extraction with Machine Learning", International Conference on Intelligent Computing and Control Systems ICICCS", pp. 1048-1053, 2017.

[45] A. K. Jain and A. Vailaya, "Image Retrieval using Color and Shape," Pattern Recognit., vol. 29, pp. 1233-1244, 1995.

[46] R. Zhao and W. I. Grosky, "From features to semantics: some preliminary results," Multimed. Expo, 2000. ICME 2000. 2000 IEEE Int. Conf., vol. 2, no. c, pp. 679-682 vol.2, 2000. 
[47] R. Chaudhari and A. M. Patil, "Content Based Image Retrieval Using Color and Shape Features," Int. J. Adv. Res. Electr. Electron. Instrum. Eng., vol. 1, no. 5, pp. 386-392, 2012.

[48] L. C. Siong, W. M. D. W. Zaki, A. Hussain, H. A. Hamid, and H. A. Hamid, "Image Retrieval System for Medical Applications," pp. 73-77, 2015.

[49] Apostolos Marakakis et al., "Relevance Feedback for Content Based Image Retrieval Using Support Vector Machines and Feature Selection”, Springer, ICANN 2009, Part I, LNCS 5768, pp. 942-951, 2009.

[50] Pengyu Hong, Qi, Tian, Thomas S. Huang, "Incorporate Support Vector Machines to Content Based Image Retrieval with Relevance Feedback" Proc. IEEE 2000 International Conference on Image Processing (ICIP 2000), pp. 750-753, Vol.3, 2000.

*Corresponding author.

E-mail address: priyankasaxena1993@yahoo.com/ munjalshefali@ gmail.com 